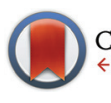

CrossMark

\& click for updates

Cite this: Food Funct., 2015, 6, 730

Received 26th January 2015,

Accepted 4th February 2015

DOI: 10.1039/c5fo00099h

www.rsc.org/foodfunction

\title{
Effect of substituent pattern and molecular weight of cellulose ethers on interactions with different bile salts
}

\author{
Amelia Torcello-Gómez, ${ }^{* a}$ Cristina Fernández Fraguas, ${ }^{b}$ Mike J. Ridout, ${ }^{b}$ \\ Nicola C. Woodward, ${ }^{\text {b }}$ Peter J. Wilde ${ }^{b}$ and Timothy J. Foster ${ }^{a}$
}

\begin{abstract}
Some known mechanisms proposed for the reduction of blood cholesterol by dietary fibre are: binding with bile salts in the duodenum and prevention of lipid absorption, which can be partially related with the bile salt binding. In order to gain new insights into the mechanisms of the binding of dietary fibre to bile salts, the goal of this work is to study the main interactions between cellulose derivatives and two types of bile salts. Commercial cellulose ethers: methyl (MC), hydroxypropyl (HPC) and hydroxypropylmethyl cellulose (HPMC), have been chosen as dietary fibre due to their highly functional properties important in manufactured food products. Two types of bile salts: sodium taurocholate (NaTC) and sodium taurodeoxycholate (NaTDC), have been chosen to understand the effect of the bile salt type. Interactions in the bulk have been investigated by means of differential scanning calorimetry (DSC) and linear mechanical spectroscopy. Results show that both bile salts have inhibitory effects on the thermal structuring of cellulose ethers and this depends on the number and type of substitution in the derivatised celluloses, and is not dependent upon molecular weight. Concerning the bile salt type, the more hydrophobic bile salt (NaTDC) has greater effect on these interactions, suggesting more efficient adsorption onto cellulose ethers. These findings may have implications in the digestion of cellulose-stabilised food matrices, providing a springboard to develop new healthy cellulose-based food products with improved functional properties.
\end{abstract}

\section{Introduction}

There is an increasing interest in the development of healthier foods. In this regard, the incorporation of dietary fibre in food (fibre-rich processed foods) to improve nutritional properties is one of the many possibilities. ${ }^{1,2}$ On the one hand, the food industry can take advantage of the physicochemical properties of dietary fibre to improve the viscosity, texture, sensory characteristics and shelf-life of their products. On the other hand, dietary fibre can be used as fat or sugar replacer to achieve healthier properties in food products. Furthermore, dietary fibre per se has metabolic and physiological effects in the gastrointestinal tract which can be considered as desirable. ${ }^{3}$ They are known to lower blood cholesterol. ${ }^{4,5}$ This effect is explained by the binding of dietary fibre with bile salts in the duodenum, which are sequestered and eventually

${ }^{a}$ Division of Food Sciences, School of Biosciences, University of Nottingham, Sutton Bonington Campus, Loughborough, LE12 5RD, UK.

E-mail: amelia.torcello_gomez@nottingham.ac.uk; Fax: +44(0)115 951 6142; Tel: +44 (0)1159516197

${ }^{b}$ Institute of Food Research, Norwich Research Park, Norwich, NR4 7UA, UK excreted. ${ }^{6}$ Alternatively, the prevention of lipid absorption is proposed, ${ }^{7-9}$ which can be partially related to the sequestration of bile salt due to binding. Bile salt sequestration reduces bile re-absorption, hence inducing the synthesis of bile salts from blood cholesterol to restore the content lost. ${ }^{10,11}$ However, the way in which bile salts interact with dietary fibre depends on the nature of the dietary fibre. Previous work in literature reported on electrostatic interactions between chitosan and a bile salt, ${ }^{12}$ dynamic molecular contact of beta-glucan with a bile salt and entrapment of bile micelles by an arabinoxylan matrix without direct molecular interaction. ${ }^{13}$ Due to the large variety of dietary fibre, a systematic approach should be taken to identify the dominant mechanism for each specific type of dietary fibre to interact with the bile salts, since these mechanisms remain unclear. In a very recent study, we evaluated the interactions between commercial cellulose ethers and a bile salt. ${ }^{14}$ These cellulose derivatives were chosen as a model of non-ionic dietary fibre, where the hydrophobicity can be tuned by varying the number, type and pattern of substituents. Namely these cellulose ethers are obtained by partially reacting the hydroxyl groups in sugar rings of the native cellulose backbone. Fig. 1a illustrates the cellulose ethers studied: methyl- 


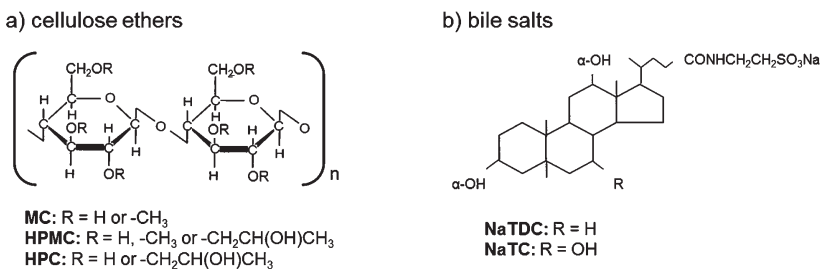

Fig. 1 Molecular structure of (a) cellulose ethers and (b) bile salts.

cellulose (MC), in which the methyl group is the sole substituent, hydroxypropylmethylcellulose (HPMC) in which the methyl group remains the dominant substituent, but incorporating smaller amounts of the larger and more polar hydroxypropyl group, and hydroxypropylcellulose (HPC) where the hydroxypropyl group is the sole substituent. In this study, hydrophobic interactions were postulated to take place between cellulose ethers and the bile salt, which were reflected in the inhibition of the thermal structuring of cellulose ethers by the bile salt. ${ }^{14}$ Differences were found regarding the number and type of substituent of the cellulose ethers. Their interfacial activity in the presence of the bile salt was also tested, showing competition for the oil-water interface. By using surface tension technique, it was also found in another recent study that ethylhydroxyethyl cellulose (EHEC) forms complexes with a bile salt in solution. ${ }^{15}$

Less attention has been paid on the type of bile salt. Bile salts are biological surfactants secreted within the duodenum, which play an important role in digestion and absorption of nutrients. The physiology of bile salts is related to their facial amphiphilic nature, ${ }^{16}$ given by a rigid steroid backbone containing methyl groups that are oriented to a hydrophobic face, whereas the hydroxyl groups and the amino group are oriented to a hydrophilic face. Bile salts differ in the number, position, and stereochemistry of the hydroxyl groups as well as the conjugated amino acid. Despite their large variety, bile salts behave in a qualitative similar manner. ${ }^{17}$ However, they also behave quantitatively different. It has been shown that their amphiphilicity is strongly influenced by subtle variations in the molecular structure, ${ }^{18}$ leading to different interfacial activity on hydrophobic interfaces. ${ }^{19-21}$ According to Armstrong and Carey (1982), the molecular hydrophobicity of bile salts depends on the cholate and conjugation group. ${ }^{18}$ The hydrophobicity decreases in the following order regarding the cholate group: deoxycholate $>$ chenodeoxycholate $>$ cholate $>$ ursodeoxycholate. Regarding the conjugation, the hydrophobicity decreases as: free bile salts $>$ glycine-conjugates $>$ tauro- conjugates. Sodium taurodeoxycholate (NaTDC) and sodium taurocholate (NaTC) were used to test the effect of hydrophobicity of the bile salt type in the present study. Being both tauro-conjugates, the only difference lies on the cholate conjugation group (Fig. 1b). Hence, NaTDC is more hydrophobic than NaTC.

A better understanding of the mechanisms whereby dietary fibres interact/bind to bile salts in the duodenum with the consequent cascade of events, such as reduction in fat absorption and blood cholesterol, can be exploited in tailoring both novel food and pharmacological matrices with improved functional properties.

\section{Materials and methods}

\subsection{Materials}

METHOCEL $^{\text {TM }}$ A4M, E4M and K4M were kindly provided by the Dow Chemical Company. The initial letter ' $\mathrm{A}$ ' denotes MC, ' $\mathrm{E}$ ' and ' $\mathrm{K}$ ' corresponds to HPMC. In each case, ' $4 \mathrm{M}$ ' denotes a solution viscosity of $4000 \mathrm{mPa} \cdot \mathrm{s}$, measured at $2 \mathrm{wt} \%$ concentration under standard conditions in a capillary viscometer. Klucel ${ }^{\circledR}$ HPC HF was purchased from Hercules. Two HPMCs from Sigma-Aldrich were also tested: H8384 and H9262, which correspond to the low-molecular weight $(\mathrm{Mw})$ homologues of E4M and K4M, respectively. The different levels of incorporation of the two substituents and range of viscosities are indicated in Table 1.

The bile salts used in this study are sodium taurodeoxycholate (NaTDC, $\geq 97 \%$ purity) and sodium taurocholate (NaTC, $\geq 97.0 \%$ purity) from Sigma-Aldrich. They are negatively charged at pH 7 and their molecular weights are 521.69 and $537.68 \mathrm{Da}$, respectively.

The buffer used in all solutions was $1.13 \mathrm{mM}$ phosphate $(\mathrm{pH} 7)$ prepared with ultrapure water purified in a Pur1te Select system. The effect of buffer was probed by using also $2 \mathrm{mM}$ BIS-TRIS (Sigma-Aldrich, $\geq 99.0 \%$ purity) adjusted to pH 7 with HCl. No differences between buffer types were observed in the results. All the chemicals were used as received without further purification.

\subsection{Sample preparation}

Cellulose ether stock solutions were prepared at $2 \mathrm{wt} \%$ as follows. Approximately one-third of the required final volume of buffer was heated to $\sim 80{ }^{\circ} \mathrm{C}$, and then cellulose ether powder was added carefully under stirring. The complete solubilisation was obtained by adding the remaining volume of

Table 1 Physicochemical characteristics of the cellulose ethers

\begin{tabular}{|c|c|c|c|c|c|c|c|c|}
\hline $\mathrm{MC}$ & $\mathrm{A} 4 \mathrm{M}$ & & $\begin{array}{l}\text { Methoxyl } \\
(\%)\end{array}$ & $\begin{array}{l}\text { Hydroxypropyl } \\
(\%)\end{array}$ & 4000 & & $300-500$ & \\
\hline & $\mathrm{K} 4 \mathrm{M}$ & H9262 & $19-24$ & $7-12$ & & & & \\
\hline HPC & $\mathrm{HF}$ & & 0 & 75 & $1500-3$ & $\mathrm{wt} \%)$ & 1115 & \\
\hline
\end{tabular}


buffer at room temperature and continuing the agitation of the solution for at least $2 \mathrm{~h}$ at room temperature. Solutions were then stored at $4{ }^{\circ} \mathrm{C}$ overnight to achieve the maximum hydration, and without stirring to eliminate air bubbles. Aliquots from the stock solutions were dried at $50{ }^{\circ} \mathrm{C}$ overnight to confirm that the final concentration was $2 \mathrm{wt} \%$.

Bile salts stock solutions were prepared by dissolving the powder in buffer at room temperature under stirring for at least $1 \mathrm{~h}$.

Different concentrations of cellulose ethers and bile salts were obtained by successive dilution in buffer from the concentrated stock at room temperature.

Mixed cellulose ether-bile salt solutions were prepared from aliquots of cellulose ethers and bile salts solutions at room temperature, in various proportions. The final concentration of cellulose ethers was fixed at $1 \mathrm{wt} \%$, whereas the final concentration of bile salts ranged from 0 to $100 \mathrm{mM}$.

\subsection{Micro-differential scanning calorimetry (micro-DSC)}

The thermal events of cellulose ethers in the absence and presence of bile salts were characterised in a Micro-Differential Scanning Calorimeter (DSC III Setaram, Caluire, France). Approximately $800 \mathrm{mg}$ of each sample were sealed into the DSC cells made from Hastalloy. The reference cell was filled with the same weight of buffer and coordinated for heat capacity with the sample. Sample and reference cells were initially cooled to a starting temperature of $5^{\circ} \mathrm{C}$ at $1^{\circ} \mathrm{C} \mathrm{min}^{-1}$ to equilibrate. Cells were then run at a scanning rate of $1{ }^{\circ} \mathrm{C}$ $\min ^{-1}$ from 5 to $110^{\circ} \mathrm{C}$, cooled and rerun while all steps were recorded. The heating/cooling rate was selected as a compromise between the need for proximity to the equilibrium (heating rate as low as possible) and an acceptable signal/disturbance ratio (heating rate not too low). Enthalpy values were calculated using Setaram software with a linear interpolated baseline, based on an extension of the trace before and after the thermal event.

\subsection{Linear mechanical spectroscopy}

Rheological measurements were carried out using a torsional stress-controlled rheometer (Physica MCR 301, Anton Paar, Austria) working, in this case, in Direct Strain Oscillation (DSO) mode, with a serrated parallel-plate geometry of $25 \mathrm{~mm}$ diameter and $1 \mathrm{~mm}$ gap. The viscoelastic moduli were provided in the measurements: the storage modulus, $G^{\prime}$, which accounts for the elasticity and the loss modulus, $G^{\prime \prime}$, which accounts for the viscosity of the sample. The linear viscoelastic regime (LVR) was determined by means of strain amplitude sweep tests at a constant angular frequency of $10 \mathrm{rad} \mathrm{s} \mathrm{s}^{-1}$. Then, the mechanical spectra of the samples were obtained from small-amplitude frequency sweep tests where the applied strain amplitude was within the LVR. The excitation frequency ranged over at least three decades well below the onset of inertial effects. Strain amplitude and frequency sweep tests were carried out below and above the transition temperature for all the samples.
For the temperature sweep tests, the samples were loaded at $20^{\circ} \mathrm{C}$ and cooled to a starting temperature of $10^{\circ} \mathrm{C}$ at $1{ }^{\circ} \mathrm{C}$ $\min ^{-1}$ to equilibrate. Then, temperature sweep tests were carried out at a fixed angular frequency $\left(10 \mathrm{rad} \mathrm{s}^{-1}\right)$ and strain (ensuring the linearity of viscoelasticity within the temperature range) from 10 to $90{ }^{\circ} \mathrm{C}$ and cooled down to $10{ }^{\circ} \mathrm{C}$ at a scanning rate of $1{ }^{\circ} \mathrm{C} \mathrm{min}^{-1}$. In order to ensure no temperature gradients within the sample, a peltier-controlled lower plate equipped with TruGap $^{\mathrm{TM}}$ function and a peltier-controlled hood were used. In addition, to avoid evaporation during the measurements, the exposed periphery of the sample was sealed with low-viscosity mineral oil (Sigma-Aldrich, cat no. 330779). Each test was run in duplicate.

\section{Results and discussion}

This section will be divided in three independent subsections to account for the effect of the physicochemical properties of cellulose ethers and bile salts on their interactions in the aqueous phase. Namely, we will evaluate the effect of the number and type of substituent of cellulose ethers, as well as of their molecular weight, to end with the effect of bile salt type. As a reference, the bulk characterisation of cellulose ethers alone, that is, in the absence of bile salts, is also included in order to compare later on with the behaviour of mixed systems: cellulose ethers/bile salts.

\subsection{Effect of substituent type/pattern of cellulose ethers}

The first part of this study is focused on identifying the effect of number and type of substituent of cellulose ethers on their thermal structuring in the presence of bile salts. Fig. 2-4 display viscoelastic moduli (top graphs) and micro-DSC thermograms (bottom graphs) upon heating/cooling for highMw MC, HPC and HPMC, respectively, in the absence and presence of the bile salt NaTDC. The concentration of cellulose ethers is fixed at $1 \mathrm{wt} \%$, while the bile salt concentration ranges from 0 to $100 \mathrm{mM}$. Fig. 2 and 3 will be discussed first since MC and HPC contain one type of substituent each, either methyl or hydroxypropyl, respectively. This will provide, in Fig. 4, a better understanding of the behaviour of HPMC that contains both types of substituent at different ratio (Table 1).

Fig. 2 shows the results for MC. Upon heating in the absence of bile salt, both techniques reflect a thermal transition at approximately $63{ }^{\circ} \mathrm{C}$, which is indicated by an endothermic peak in the micro-DSC traces (Fig. 2, bottom left, solid line) and a change in the slope of both viscoelastic moduli located at this temperature (Fig. 2, top left, diamonds). This transition corresponds to the thermal gelation of MC. ${ }^{22-24}$ It is interesting to note that the storage, $G^{\prime}$, and loss, $G^{\prime \prime}$, moduli start to increase before reaching the transition temperature. According to previous work, ${ }^{22} \mathrm{MC}$ is found in solution at room temperature as bundles where strands are held together by packing of unsubstituted regions and by hydrophobic clustering of methyl groups in regions of denser substi- 

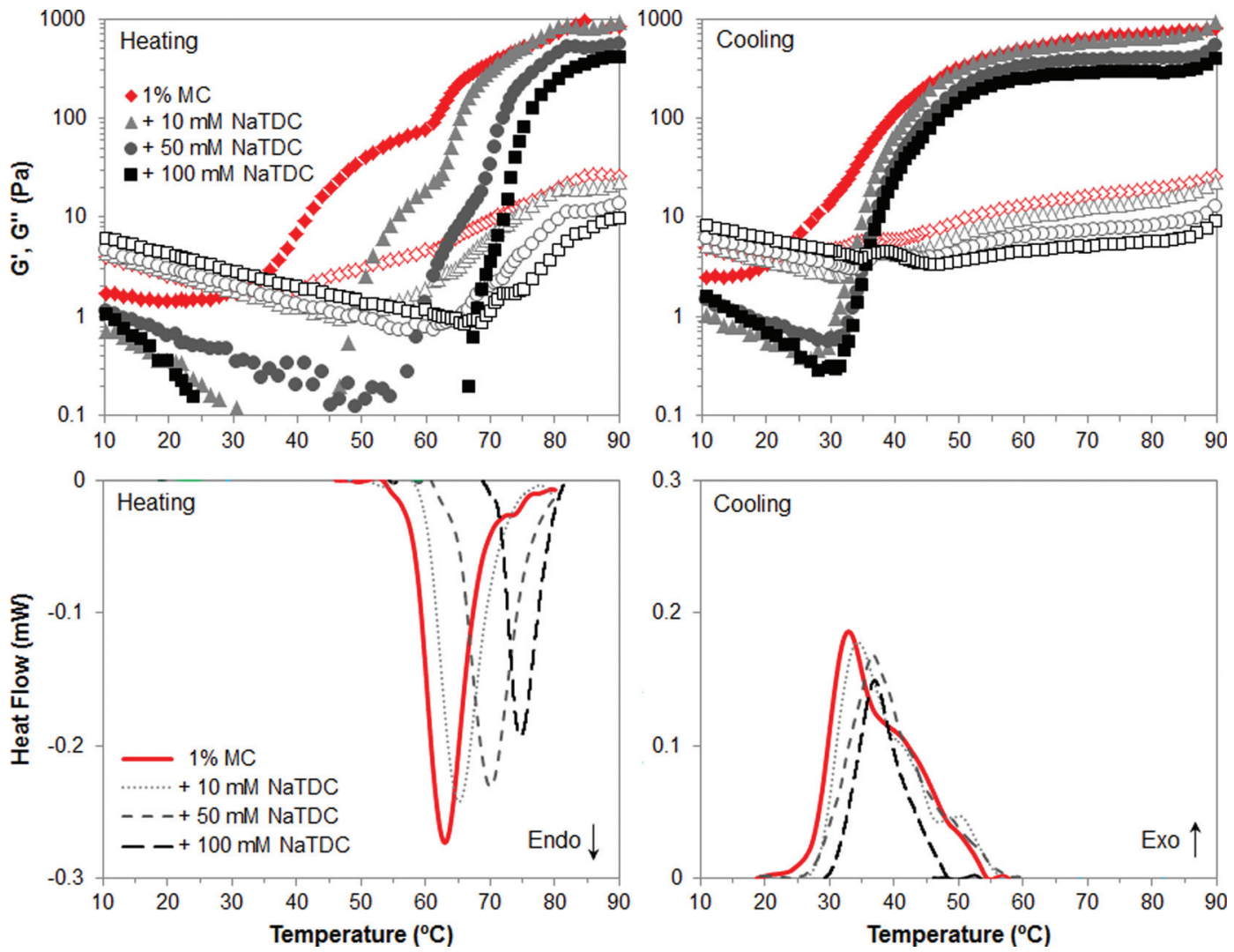

Fig. 2 Viscoelastic moduli (top; closed symbols: $G^{\prime}$; open symbols: $\left.G^{\prime \prime}\right)$ and micro-DSC traces (bottom) of 1 wt\% $M C$ upon heating/cooling (1 ${ }^{\circ} \mathrm{C}$ $\mathrm{min}^{-1}$ ), in the absence and presence of the bile salt NaTDC.

tution. In a first step upon heating, the strands separate at the ends of the bundles and methyl groups are exposed. Hence, the swelling of the bundles is reflected in the first increase of $G^{\prime}$ and $G^{\prime \prime}{ }^{22}$ The exposure of methyl groups allows the second step to take place when reaching the gelation temperature, that is, the association of hydrophobic regions of strands from different bundles, forming a physical network. Then, upon cooling, the gel disassociates, which is reflected in the hightemperature shoulder of the exothermic peak (Fig. 2, bottom right, solid line) and the onset of the decrease of the storage modulus (Fig. 2, top right, diamonds). ${ }^{22}$ Upon further decreasing the temperature, swollen bundles recover the original structure in solution, which corresponds to the main exothermic peak and change in slope of $G^{\prime}$. In addition, a clear thermal hysteresis is evident upon heating/cooling. Next, the presence of the bile salt NaTDC in MC solution increases the transition temperature upon heating, which is observed in the shift of both micro-DSC peaks and change in the slope of viscoelastic moduli. The higher the bile salt concentration, the greater the shift of the thermal transition. Moreover, NaTDC decreases the gel viscoelasticity since $G^{\prime}$ and $G^{\prime \prime}$ reach lower values above the transition temperature, and also reduces the transition enthalpy, calculated from the area below the microDSC peaks. Furthermore, it can be seen that the first wave of increase in $G^{\prime}$ and $G^{\prime \prime}$ is reduced to a narrower temperature range as bile salt concentration increases. This suggests that the bile salt hinders the swelling stage of cellulose bundles prior to gelation, although the network is subsequently formed upon further increasing the temperature, regardless of the bile salt concentration. In a recent study, we postulated that the bile salt would adsorb at low temperatures to the methyl groups of cellulose ethers in the regions of denser substitution. ${ }^{14}$ Upon heating, the newly exposed methyl groups would still be available when the strands separate at the end of the bundles, in order to form the hydrophobic association of strands from different bundles. Bile salts appear to affect the swelling stage prior to gelation and may compete, upon heating, for the available methyl groups, interfering with their hydrophobic association between different bundles. We propose that this gives rise to a weaker MC gel, as observed in lower values of $G^{\prime}$ and $G^{\prime \prime}$ at high-temperature plateaus and transition enthalpy, as compared to $\mathrm{MC}$ alone. These new findings importantly complement our previous work. ${ }^{14}$ The disruption of the gel properties at high temperature is significantly noticeable at a bile salt concentration of $50 \mathrm{mM}$, much higher than the critical micelle concentration (CMC) of NaTDC at $20{ }^{\circ} \mathrm{C}$ under the same ionic strength and $\mathrm{pH}$ conditions $(\mathrm{CMC}=3-10 \mathrm{mM}) \cdot{ }^{25}$ Hence, bile salt micelles may be playing a role in these interactions with the polymer network. On cooling however, the temperature of gel dissociation in the presence of the bile salt does not differ from that in the absence of the bile salt. That is, the high-temperature shoulder 

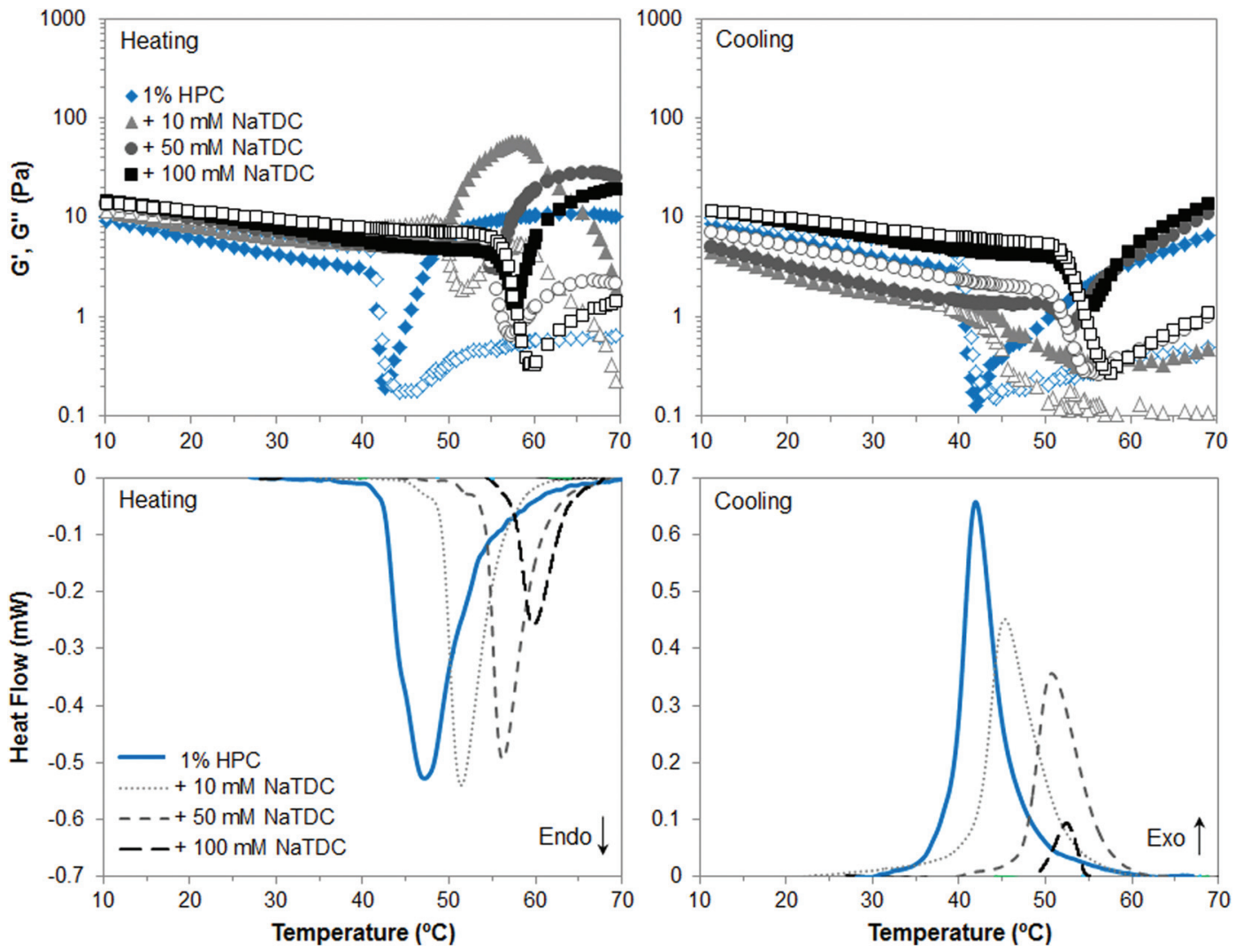

Fig. 3 Viscoelastic moduli (top; closed symbols: $G^{\prime}$; open symbols: $\left.G^{\prime \prime}\right)$ and micro-DSC traces (bottom) of 1 wt\% HPC upon heating/cooling (1 ${ }^{\circ} \mathrm{C}$ $\mathrm{min}^{-1}$ ), in the absence and presence of the bile salt NaTDC.

of the exothermic peak and the onset of the decrease of the storage modulus are located at similar temperature, regardless of the bile salt concentration. Nevertheless, upon further decreasing the temperature, we can observe that the second process whereby the swollen bundles recover the original structure in solution, corresponding to the second wave of $G^{\prime}$ decrease, is confined again to a narrower temperature range induced by the bile salt. This is reflected in the slight shift of the main exothermic peaks to higher temperature as the bile salt concentration increases and less appreciable change in the slope of $G^{\prime}$. Hence, the presence of the bile salt seems to promote the recovery of the bundles to the original structure in solution.

In the case of HPC, Fig. 3 shows again that, in the absence of bile salt, both techniques reveal upon heating a transition point at approximately $47^{\circ} \mathrm{C}$, which corresponds to a "thermal demixing". ${ }^{26}$ This process does not involve proper gelation. Since HPC is highly substituted (Table 1), the more "uniform" distribution pattern of hydroxypropyl groups along the polymer chains prevent them from packing at low temperature in contrast to MC. Upon heating, the hydrophobic association takes place, which is called "thermal demixing", giving rise to the onset of the endothermic peak in micro-DSC thermograms (Fig. 3, bottom left, solid line) and to a drastic drop of viscoelastic moduli (Fig. 3, top left, diamonds). Then, as hydrophobic clusters are formed upon further increasing temperature, $G^{\prime}$ and $G^{\prime \prime}$ increase and level, corresponding to the offset of the endothermic peak, but a physical network is not formed. This process is reversible upon cooling as observed in the exothermic peak (Fig. 3, bottom right, solid line) and recovery of viscoelastic moduli (Fig. 3, top right, diamonds), with slight thermal hysteresis, as compared to gelation of MC. ${ }^{14,26}$ Sedimentation of the hydrophobic aggregates may take place over time, ${ }^{27}$ which is reflected in lower $G^{\prime}$ and $G^{\prime \prime}$ on cooling than on heating, before complete recovery of HPC solution at low temperature. Next, the presence of bile salt increases the transition temperature and reduces the transition enthalpy, as NaTDC concentration increases. In this case the effect of the bile salt is already noticeable at a bile salt concentration of $10 \mathrm{mM}$. This may be due to the polymer hydrophobicity and distribution pattern of the hydrophobic moieties. In the case of HPC, the more "uniform" distribution of bile salt along the polymer chains through its adsorption onto hydroxypropyl groups would hinder to a larger extent the hydrophobic association upon heating, as compared to MC. ${ }^{14}$ The sedimentation of hydrophobic aggregates is very obvious in the mixture of HPC and $10 \mathrm{mM}$ NaTDC, since $G^{\prime}$ and $G^{\prime \prime}$ reach a maximum before decreasing already on heating, at temperatures corresponding to the offset of the endotherm trace, when the transition is almost complete. After clusters formation, $G^{\prime}$ and $G^{\prime \prime}$ are higher in the presence of the bile salt, regardless of the concentration, which might be ascribed 

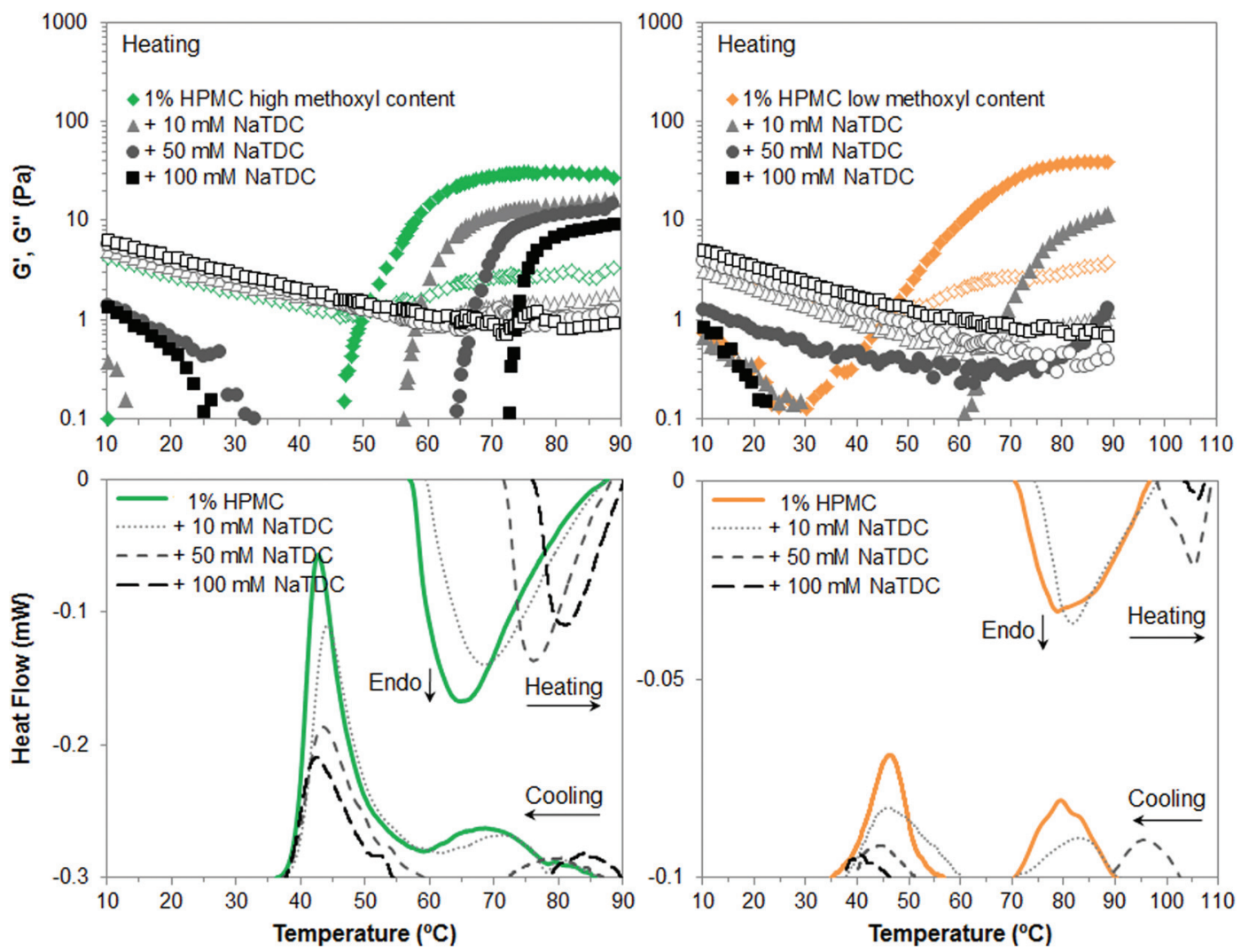

Fig. 4 Viscoelastic moduli (top; closed symbols: $G^{\prime}$; open symbols: $\left.G^{\prime \prime}\right)$ and micro-DSC traces (bottom) of 1 wt\% HPMC upon heating/cooling (1 ${ }^{\circ} \mathrm{C}$ $\mathrm{min}^{-1}$ ), in the absence and presence of the bile salt NaTDC.

to hydrophobic aggregates of larger size formed by mixed HPC and bile salt.

Finally, Fig. 4 illustrates the gelation behaviour of HPMC, which in the absence of bile salt is qualitatively similar to that of MC although forming a weaker gel, as reflected in lower viscoelastic moduli for both types of HPMC (Fig. 4, top, diamonds) and lower transition enthalpies in the case of lowmethoxyl content HPMC (Fig. 4, bottom right, solid lines). This is attributed not only to the lower content of methyl groups (Table 1) but also to the presence of the more polar hydroxypropyl groups, which shift the gelation process to higher temperatures as compared to MC. The lower the methoxyl content, the more polar the HPMC and the greater the shift in temperature. The presence of a high-temperature exotherm on cooling was first observed in our previous study with the low-methoxyl content HPMC. ${ }^{14}$ Our findings are now confirmed for the high-methoxyl content HPMC in Fig. 4. This contrasts with the broad single exothermic peaks reported earlier for both HPMCs. ${ }^{26}$ Slight variations in the degree of substitution and pattern of distribution for each substituent may be found for different batches of these commercial samples, which may lead to a different thermal behaviour. Hence, this exothermic peak is tentatively attributed to possible distributions of substituents on the cellulose bundles. ${ }^{28}$ Next, the addition of the bile salt to HPMC solutions again has similar effect as for MC and HPC. Nonetheless, differences are observed regarding the methoxyl content. Namely, NaTDC weakens to a larger extent the gel formed by HPMC of lower methoxyl content. This is observed in appreciably lower viscoelastic moduli above the transition temperature at the lowest bile salt concentration (10 $\mathrm{mM})$ for low-methoxyl HPMC (Fig. 4, top right, triangles), as compared to high-methoxyl HPMC (Fig. 4, top left, triangles). Also, increasing the bile salt concentration greatly increases the transition temperature and reduces the transition enthalpy for low-methoxyl HPMC (Fig. 4, bottom right) in contrast to high-methoxyl content HPMC (Fig. 4, bottom left). The gel strength of low-methoxyl HPMC could not be measured since the transition in the presence of bile salt at 50 and $100 \mathrm{mM}$ concentration takes place above the temperature range tested in the rheometer. Nonetheless, it is worth noting that a $100 \mathrm{mM}$ bile salt concentration practically inhibits the thermogelation of low-methoxyl content HPMC, as seen from the minimal peaks (long-dashed lines in Fig. 4, bottom right) detected by calorimetry upon heating/cooling. We think that the adsorption of bile salt onto the larger hydroxypropyl groups would hinder to a larger extent the thermal structuration due to steric effects, in the same way that hydroxypropyl groups are "difficult" to incorporate within ordered structures. ${ }^{26}$ Therefore, by reducing the methoxyl content in the HPMC, the hydroxypropyl percentage increases with respect to the total content of substituent groups, and consequently the effect of the bile salt is greater. 

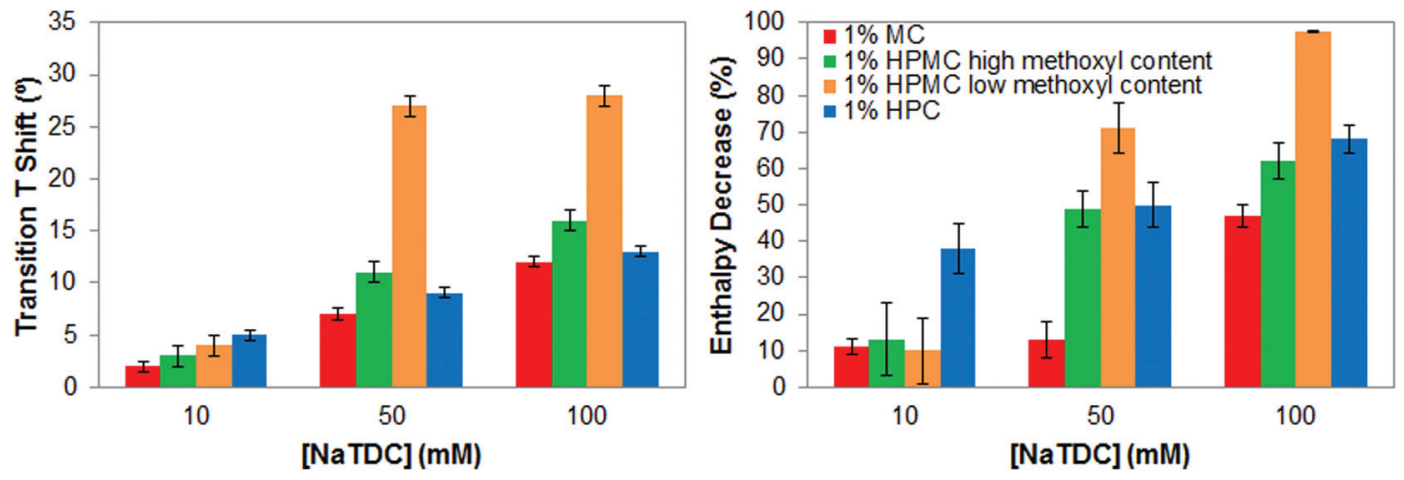

Fig. 5 Increase in transition temperature (left) and transition enthalpy reduction (right), of 1 wt\% cellulose ethers upon heating caused by the bile salt NaTDC.

To better ascertain differences regarding the type of substituent and pattern of distribution, Fig. 5 summarises the increase of the transition temperature and the transition enthalpy reduction of cellulose ethers as the bile salt concentration increases, that is, as the ratio bile salt/cellulose ether increases. As seen before, the increase of the transition temperature and the decrease of the transition enthalpy is proportional to the ratio bile salt/cellulose ether. ${ }^{14}$ However, the effect of the bile salt is greater when incorporating hydroxypropyl groups (HPC and HPMC) in the cellulose ethers and in the case of HPMC when reducing the methoxyl content. Thus, the presence of bile salt seems to better disrupt the thermal transition of cellulose ethers when it is adsorbed onto the larger hydroxypropyl groups, as suggested previously. Ultimately, the lower degree of substitution and lower methoxyl/hydroxypropyl ratio in HPMC is crucial for visualising the most extreme effect of the bile salt on gelation properties of cellulose ethers. The thermal structuring is almost completely inhibited by the highest bile salt concentration. Therefore, the interactions between cellulose ethers and the bile salt importantly depend on the type of substituent and pattern of distribution.

\subsection{Effect of the molecular weight of cellulose ethers}

We will next evaluate whether the molecular weight of cellulose ethers, specifically of HPMC, has a relevant effect on their interactions with bile salts. This is of interest since it might have an impact during the digestion of cellulose-stabilised food matrices. Fig. 6 compares the increase in the transition temperature and the enthalpy reduction of high- and low-Mw HPMC of high and low methoxyl content, respectively, caused by NaTDC as a function of the bile salt concentration. Fig. 6 shows that the trend in the increase of the transition temperature and in the enthalpy reduction caused by the bile salt is similar for high- and low-Mw HPMC, regardless of the methoxyl content. Namely, low-methoxyl content HPMCs exhibit larger increase in gelation temperature and greater enthalpy reduction than high-methoxyl content HPMCs, as the bile salt concentration increases from 10 to $100 \mathrm{mM}$. This agrees with the effect of the type/pattern of substituent seen in section 3.1. Nonetheless, there is not a clear tendency regarding the molecular weight. For some of the mixtures of HPMC and NaTDC the values of change in transition temperature and enthalpy are similar within the margin of error. We attribute then variations in the results between the high-and low-Mw HPMC to possible differences in the substituent content/distribution since the samples come from different suppliers.

On the other hand, Fig. 7 displays the mechanical spectra of high- and low-Mw HPMCs as the bile salt concentration increases. The frequency sweep tests were performed $12{ }^{\circ} \mathrm{C}$ above the gelation temperature for each mixture of HPMC and
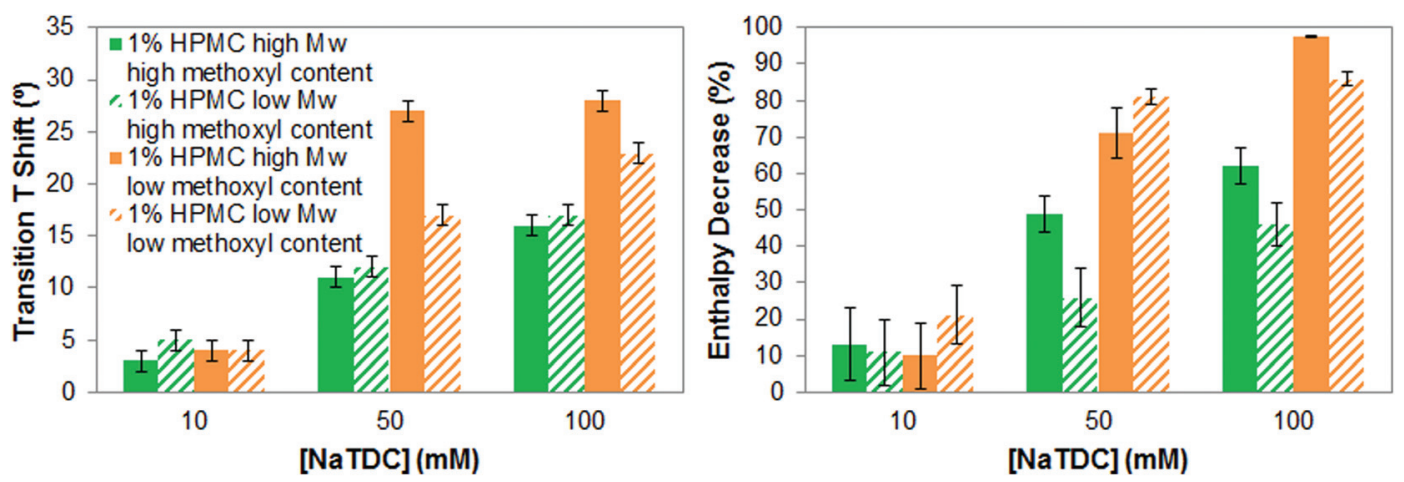

Fig. 6 Increase in transition temperature (left) and transition enthalpy reduction (right), of 1 wt\% high- and low-Mw HPMC upon heating caused by the bile salt NaTDC. 

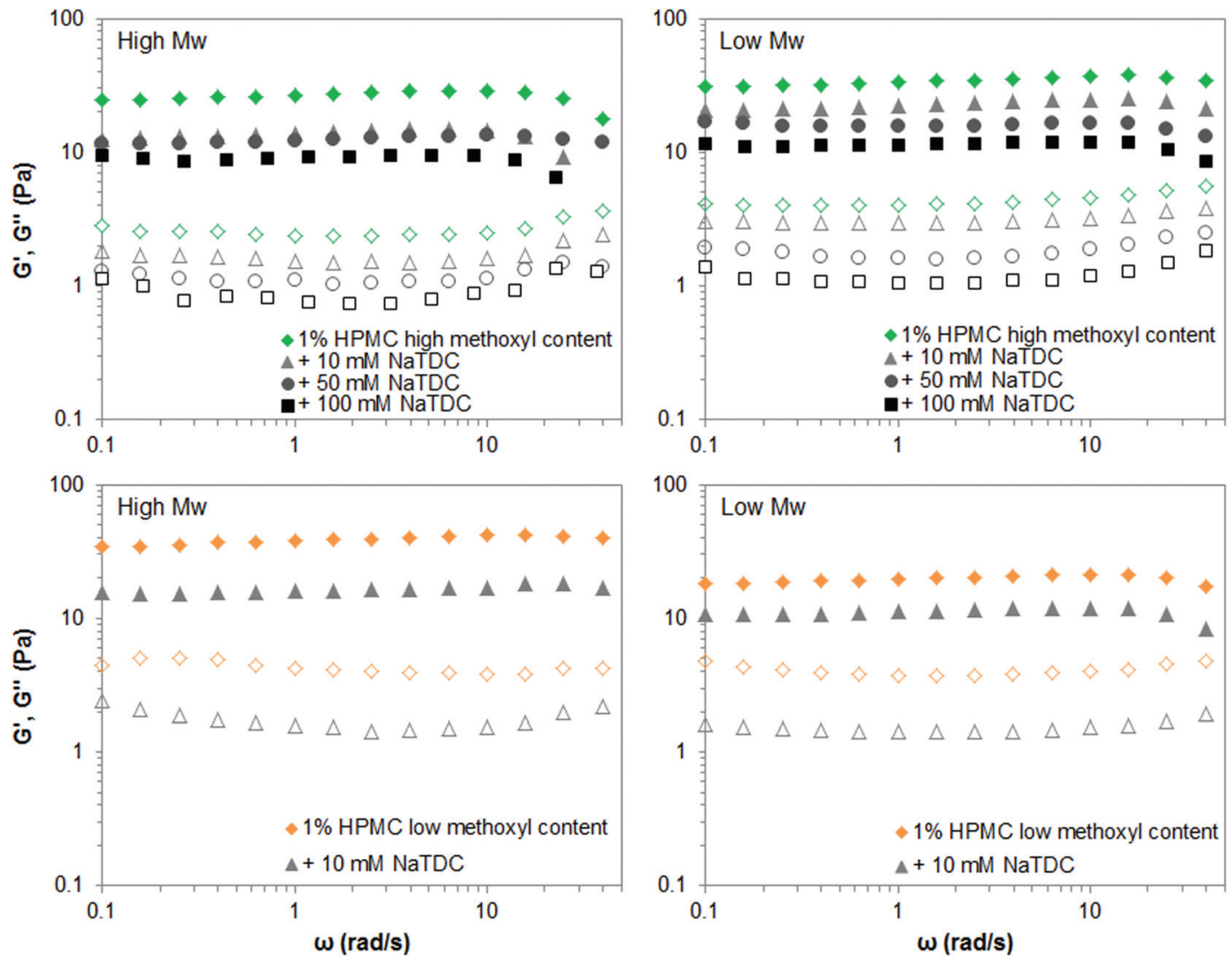

Fig. 7 Mechanical spectra (closed symbols: $G^{\prime}$; open symbols: $G^{\prime \prime}$ ) of 1 wt\% high- and low-Mw HPMC above the transition temperature, in the absence and presence of the bile salt NaTDC.

NaTDC, when a plateau is reached on the viscoelastic moduli upon heating (like in Fig. 4). Fig. 7 shows that NaTDC reduces the viscoelastic moduli to similar extent for both high- and low-Mw HPMCs, regardless of the methoxyl content. This supports the results from Fig. 6. Again, variations between the high-and low-Mw HPMC may be due to differences in the substituent content/distribution. It is also worth noting that the bile salt greatly disrupts the viscoelastic properties of the lowMw HPMC of low methoxyl content, as seen by a drastic reduction of $G^{\prime}$ and $G^{\prime \prime}$ at the lowest concentration of $10 \mathrm{mM}$ studied here. Higher bile salt concentrations inhibit the gel formation, as observed also from calorimetry results, where NaTDC causes a substantial reduction of the enthalpy transition and a great shift of the gelation to higher temperatures (Fig. 6) similarly as for high-Mw homologue (Fig. 4 and 5). Therefore, the corresponding frequency sweep tests could not be performed since the transition temperature was outside the temperature range measured in the rheometer. This again supports the effect of the type/pattern of substituent seen in section 3.1.

The fact that the molecular weight of HPMC does not have an important effect on its interaction with bile salts can be explained as follows. Since cellulose ethers are fibres, the area/ volume aspect ratio is not relevant, but the distribution of substituent groups per unit length. At a fixed concentration of cellulose ethers, $1 \mathrm{wt} \%$ in this case, the amount of low-Mw fibres, i.e. shorter chains, is higher than high-Mw fibres, i.e. longer chains, but the weight is the same and therefore, the substituent pattern per unit length. Thus, it seems that hydrophobicity is the main driving force for these interactions to take place. If that is the case, then the hydrophobicity of the bile salt may affect also these interactions. This will be probed in next section.

\subsection{Effect of the bile salt type}

Finally, the effect of the bile salt type on the interactions with cellulose ethers is tested by comparing the results obtained with two different bile salts, NaTDC, the one studied so far, and NaTC. Fig. 8 shows the increase in the transition temperature for all the cellulose ethers caused by the bile salts as the concentration increases. Since we have demonstrated that these interactions are independent of the molecular weight of cellulose ethers, the results are only shown for high-Mw cellulose ethers. It can be seen differences regarding the type of bile salt used. The most remarkable result is that NaTDC shifts the thermal transition of all cellulose ethers to higher temperature than NaTC, although keeping similar transition enthalpy values (data not shown). In addition, mixtures of cellulose ether and NaTDC display narrower micro-DSC peaks than the mixtures with NaTC (results not shown), being more evident for high- and low-Mw HPMC samples, regardless of 


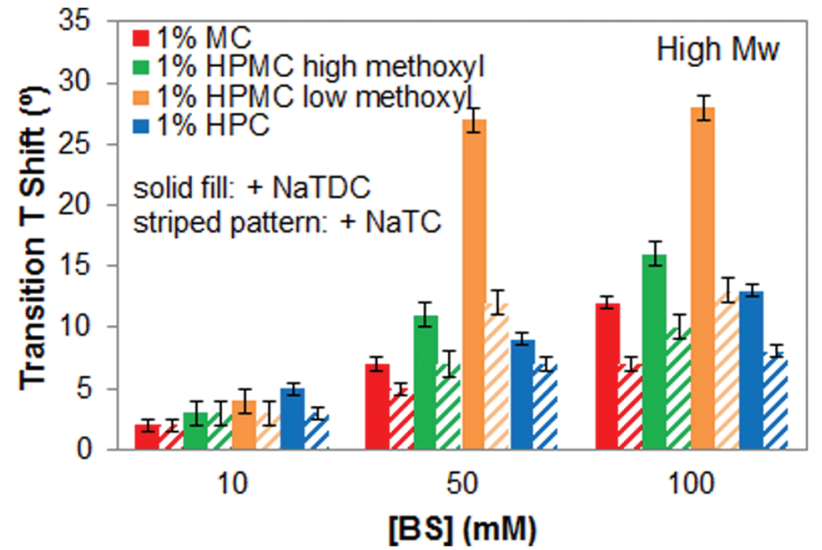

Fig. 8 Increase in transition temperature of 1 wt\% high-Mw cellulose ethers upon heating caused by either NaTDC or NaTC.

the methoxyl content. This indicates that NaTDC restricts the thermogelation of cellulose ethers to a narrower temperature range as compared to NaTC.

On the other hand, Fig. 9 shows the gel strength reduction of cellulose ethers caused by NaTC at high temperature. The frequency sweep tests were performed on cellulose ethers that gels at high temperature, that is, MC and HPMC, although temperature sweep tests were carried out for all of them, including HPC (data not shown). If we compare Fig. 9 with Fig. 2 and 4, it can be observed that NaTDC lowers the viscoelastic moduli, $G^{\prime}$ and $G^{\prime \prime}$, of MC and HPMC to a greater extent than NaTC, meaning greater disruption of their gel properties. Also, the swelling stage of the bundles, corresponding to the first wave of increase in $G^{\prime}$ and $G^{\prime \prime}$, which is more evident for MC samples, covers broader temperature range in the presence of NaTC. Therefore, as predicted above, the more hydrophobic bile salt, NaTDC has a greater effect on the thermogelation of cellulose ethers, suggesting that it adsorbs more efficiently onto the polymer chains. Interfacial studies by MaldonadoValderrama et al. showed that a deoxycholate bile salt displays more surface activity than a cholate bile salt. ${ }^{20}$ Parker and coworkers recently reported on the adsorption and desorption of bile salts to a solid hydrophobic interface, where deoxycholates also showed greater rate and extent of adsorption than cholates. ${ }^{21}$ Zhu and co-workers also showed that deoxycholates better compete for adsorption by resins than cholates. ${ }^{29}$ Therefore, this corroborates that the hydrophobicity plays a key role in these interactions. Finally, the trend of the effect of substituent type of cellulose ethers in the presence of the new bile salt, NaTC, agrees with that seen in section 3.1. Hence, both bile salts affect to a larger extent the thermal transition of the cellulose ethers containing hydroxypropyl groups and concretely of the HPMC of low methoxyl content. Also, results for low-Mw HPMCs in the presence of NaTC show the same effect as for high-Mw HPMCs (data not shown for brevity). This importantly corroborates and generalises the discussion presented in section 3.1 and 3.2 for both types of bile salt, NaTDC and NaTC.
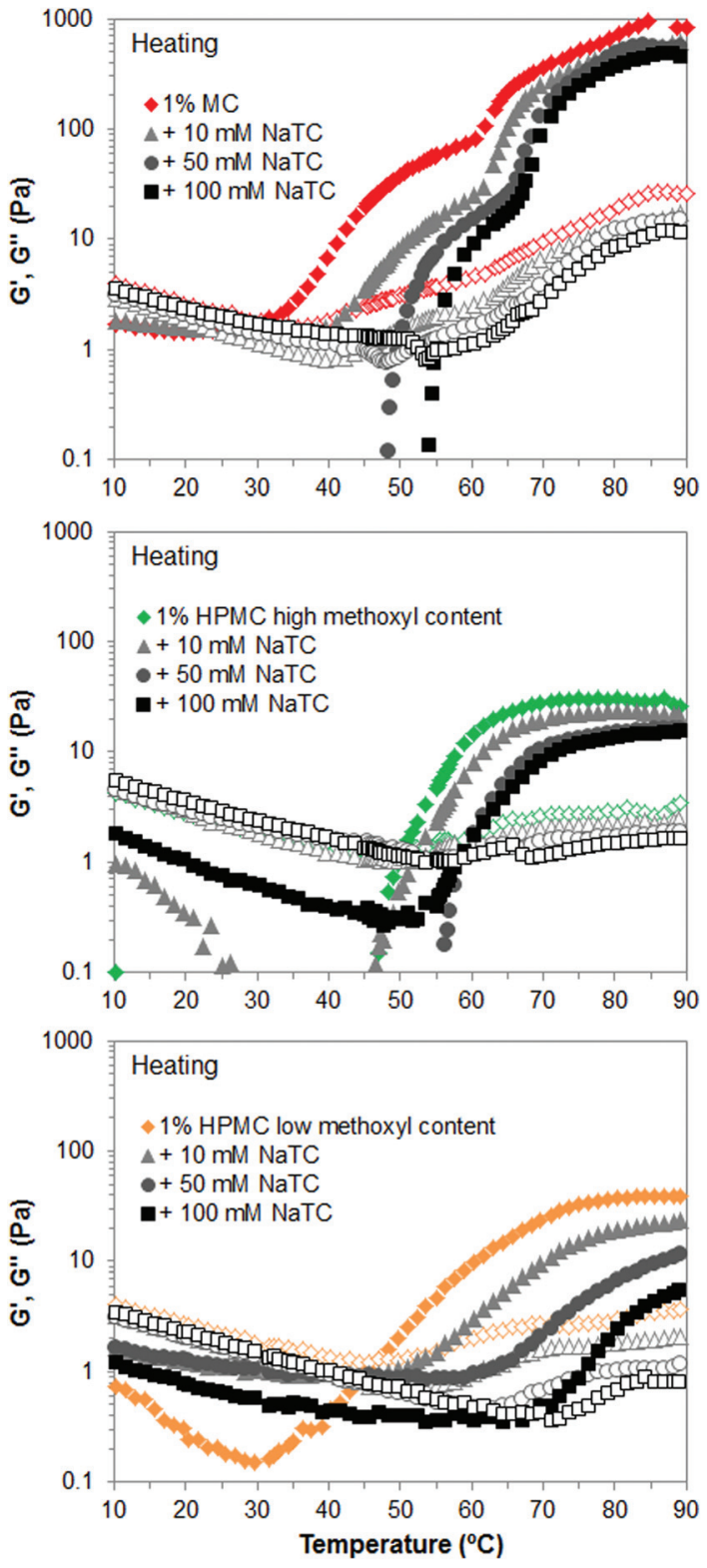

Fig. 9 Viscoelastic moduli (closed symbols: $G^{\prime}$; open symbols: $\left.G^{\prime \prime}\right)$ of 1 wt\% cellulose ethers upon heating, in the absence and presence of the bile salt NaTC.

\section{Conclusions}

Bile salts interact with cellulose ethers, as reflected in the inhibition of their thermal structuring at high temperature. Specifically, the presence of bile salts shifts the transition temperature of cellulose ethers to higher temperature, suggesting that the system is more hydrophilic. In addition, bile salts reduce the transition enthalpy of all cellulose ethers and the viscoelasticity of the gel formed by MC and HPMC. The higher the bile salt concentration, the greater the inhibition. This suggests that bile salts might adsorb at body temperature onto the hydrophobic regions of the cellulose ethers through their hydrophobic face, while exposing the hydro- 
philic face, which is experimentally revealed in the effects on thermogelation. The same rules governing the inhibition of thermal structuring would be applied for these interactions under physiological conditions.

On the one hand, the inhibitory effect depends largely on the substitution type/pattern in cellulose ethers rather than their molecular weight. Namely, the cellulose ethers containing hydroxypropyl groups (HPMC and HPC) seem more susceptible to the presence of bile salts, concretely HPMC of low methoxyl content. On the other hand, the more hydrophobic bile salt (NaTDC) might adsorb more efficiently than the more hydrophilic (NaTC) on cellulose ethers, inhibiting to a larger extent their thermogelation properties.

These findings can be exploited in tailoring both novel food and pharmacological matrices with improved functional properties.

\section{Acknowledgements}

Authors thank the financial support from the European Community's Seventh Framework Program (FP7-PEOPLE-IEF) under Grant Agreement No. 326581 and 299920.

\section{References}

1 M. Elleuch, D. Bedigian, O. Roiseux, S. Besbes, C. Blecker and H. Attia, Food Chem., 2011, 124, 411-421.

2 F. Guillon, M. Champ, J. F. Thibault and L. Saulnier, in Functional Foods: Concept to Product, ed. M. Saarela, Woodhead Publishing, 2nd edn, 2011, pp. 582-622.

3 D. L. Topping, in Encyclopedia of Human Nutrition, ed. L. H. Allen and A. Prentice, Elsevier, 3rd edn, 2013, pp. 50-54.

4 J. W. Anderson and A. E. Siesel, in New Developments in Dietary Fiber: Physiological, and Analytical Aspects, ed. I. Furda and C. J. Brine, Plenum Press, New York, 1990, vol. 270, pp. 17-36.

5 D. Kritchevsky and J. A. Story, in CRC Handbook of Dietary Fiber in Human Nutrition, ed. G. A. Spiller, CRC Press, Boca Raton, 3rd edn, 1993, pp. 163-178.

6 J. A. Story, E. J. Furumoto and K. K. Buhman, in Dietary Fiber in Health and Disease, ed. D. Kritchevsky and C. Bonfield, Plenum Press, New York, 1997, vol. 427, pp. 259-266.

7 D. Lairon, Eur. J. Clin. Nutr., 1996, 50, 125-133.

8 D. J. A. Jenkins, C. W. C. Kendall and T. P. P. Ransom, Nutr. Res., 1998, 18, 633-652.
9 W. Yokoyama, W. H. K. Anderson, D. R. Albers, Y. J. Hong, M. L. Langhorst, S. C. Hung, J. T. Lin and S. A. Young, J. Agric. Food Chem., 2011, 59, 11249-11254.

10 J. K. Lee, S. U. Kim and J. H. Kim, Biosci., Biotechnol., Biochem., 1999, 63, 833-839.

11 P. Zarras and O. Vogl, Prog. Polym. Sci., 1999, 24, 485516.

12 M. Thongngam and D. J. McClements, Food Hydrocolloids, 2005, 19, 813-819.

13 P. Gunness, B. M. Flanagan and M. J. Gidley, J. Cereal Sci., 2010, 52, 444-449.

14 A. Torcello-Gómez and T. J. Foster, Carbohydr. Polym., 2014, 113, 53-61.

15 S. D. Modolon, A. C. Felippe, T. E. Fizon, L. da Silva, M. M. D. Paula and A. G. Da-Bo, Carbohydr. Polym., 2014, 111, 425-432.

16 A. F. Hofmann and D. M. Small, Annu. Rev. Med., 1967, 18, 333-376.

17 J. Maldonado-Valderrama, P. Wilde, A. Macierzanka and A. Mackie, Adv. Colloid Interface Sci., 2011, 165, 36-46.

18 M. J. Armstrong and M. C. Carey, J. Lipid Res., 1982, 23, 70-80.

19 M. Wickham, P. Wilde and A. Fillery-Travis, Biochim. Biophys. Acta, Mol. Cell Biol. Lipids, 2002, 1580, 110122.

20 J. Maldonado-Valderrama, J. L. Muros-Cobos, J. A. Holgado-Terriza and M. A. Cabrerizo-Vílchez, Colloids Surf., B, 2014, 120, 176-183.

21 R. Parker, N. M. Rigby, M. J. Ridout, A. P. Gunning and P. J. Wilde, Soft Matter, 2014, 10, 6457-6466.

22 A. Haque and E. R. Morris, Carbohydr. Polym., 1993, 22, 161-173.

23 L. Li, H. Shan, C. Y. Yue, Y. C. Lam, K. C. Tam and X. Hu, Langmuir, 2002, 18, 7291-7298.

24 M. Tomsic, F. Prossnigg and O. Glatter, J. Colloid Interface Sci., 2008, 322, 41-50.

25 A. Torcello-Gómez, A. B. Jódar-Reyes, J. Maldonado-Valderrama and A. Martin-Rodriguez, Food Res. Int., 2012, 48, 140-147.

26 A. Haque, R. K. Richardson, E. R. Morris, M. J. Gidley and D. C. Caswell, Carbohydr. Polym., 1993, 22, 175-186.

27 A. Sullo, Y. H. Wang, A. Koschella, T. Heinze and T. J. Foster, Carbohydr. Polym., 2013, 93, 574-581.

28 S. M. Sun, T. J. Foster, W. MacNaughtan, J. R. Mitchell, D. Fenn, A. Koschella and T. Heinze, J. Polym. Sci., Part B: Polym. Phys., 2009, 47, 1743-1752.

29 X. X. Zhu, F. Brizard, J. Piche, C. T. Yim and G. R. Brown, J. Colloid Interface Sci., 2000, 232, 282-288. 